\title{
Posedness of a band-limited image-extension problem in tomography
}

\author{
Robert J. Marks II \\ Department of Electrical Engineering, FT-10, University of Washington, Seattle, Washington 98195
}

Received April 8, 1982

\begin{abstract}
The posedness of extending a band-limited image known only within a cone is considered. The problem is globally ill posed in the sense that the output-error energy cannot be bound over the entire unknown image region corresponding to a given input-data error energy. We demonstrate, however, that the problem is locally well posed in the sense that, within a finite area of the unknown image region, the error can be bound.
\end{abstract}

The following band-limited image-extension problem occurs in reconstruction of a tomographic image with limited-angle input: A two-dimensional band-limited image $u(x, y)$ is known within the shaded cone shown in Fig. 1. The extension problem is to determine the image elsewhere. We assume that we have knowledge of the image's bandwidth.

An excellent overview of the problem and possible solutions is given by Tam and Perez-Mendez. ${ }^{1}$ An iterative process for the problem solution is given by Sato et al. ${ }^{2}$

As was correctly pointed out in Ref. 1, the problem is ill posed. The inverse operator for restoration is not continuous. That is, small errors in the data can generate unbounded effective error in the solution. ${ }^{3-8}$

We can easily demonstrate that the problem is ill posed by finding a sequence of appropriately bandlimited functions that have an ever-decreasing percentage of their energy in the shaded cone approaching zero. This test, used by Wiley, ${ }^{5}$ is equivalent to demonstrating that the truncation operator norm is zero, thus prohibiting well-posed inversion. ${ }^{4,8}$ Any number of band-limited functions shifted up the $y$ axis in Fig. 1 demonstrate this property. Note also that the problem can be viewed as a continuum of one-dimensional vertical (ill-posed ${ }^{4,7}$ ) extrapolations.

The definition of ill-posedness is global. That is, error everywhere within the unshaded cone is considered. It is possible to bound the error within a specified finite area in the unshaded cone, thus demonstrating that a globally ill-posed problem can be locally well posed.

Consider the one-dimensional slice on the $(x, y)$ plane in Fig. 1, corresponding to $y=\eta$. If $u(x, y)$ is band limited, then the one-dimensional function corresponding to $u(x, \eta)$ is also band limited. ${ }^{9}$ Therefore we can apply an algorithm such as Gerchberg's ${ }^{9-13}$ to interpolate $u(x, \eta)$ within the unshaded area. Interpolation is well posed. 4,14 Thus the interpolation error can be bound for a given input-data error. The one-dimensional bandwidths of all the horizontal slices of $u(x$, $y$ ) are identical, say $\Omega_{x}{ }^{9}$ The ratio of the data-error energy to interpolation-error energy is less than (1 - $\left.\sqrt{\lambda_{0}}\right)^{-1}, 15,16$ where $\lambda_{0}$ is the largest eigenvalue of the prolate spheroidal wave-function integral equation, ${ }^{17}$

$$
\lambda_{n} \psi_{n}(x)=\Omega_{x} \int_{-a \eta}^{a \eta} \psi_{n}(\xi) \operatorname{sinc} \Omega_{x}(x-\xi) \mathrm{d} \xi,
$$

where $\operatorname{sinc}(x)=\sin (\pi x) /(\pi x)$ and $a$ is the cone parameter (see Fig. 1).

The eigenvalue $\lambda_{0}$ is bound,

$$
0<\lambda_{0}<1 \text {, }
$$

and is a monotonically increasing function of ${ }^{18}$

$$
c=\pi \Omega_{x} a \eta
$$

Thus, as expected, the output-to-input-error ratio bound $\left(1-\sqrt{\lambda_{0}}\right)^{-1}$ increases with $\eta$. Since $\lambda_{0} \rightarrow 1$ as $|\eta| \rightarrow \infty$, the global ill-posedness of the problem in this analysis arises from the error bound's growing without limit for large $|y|$. The problem, however, is locally well posed in the sense that the error in any finite area can be bound.

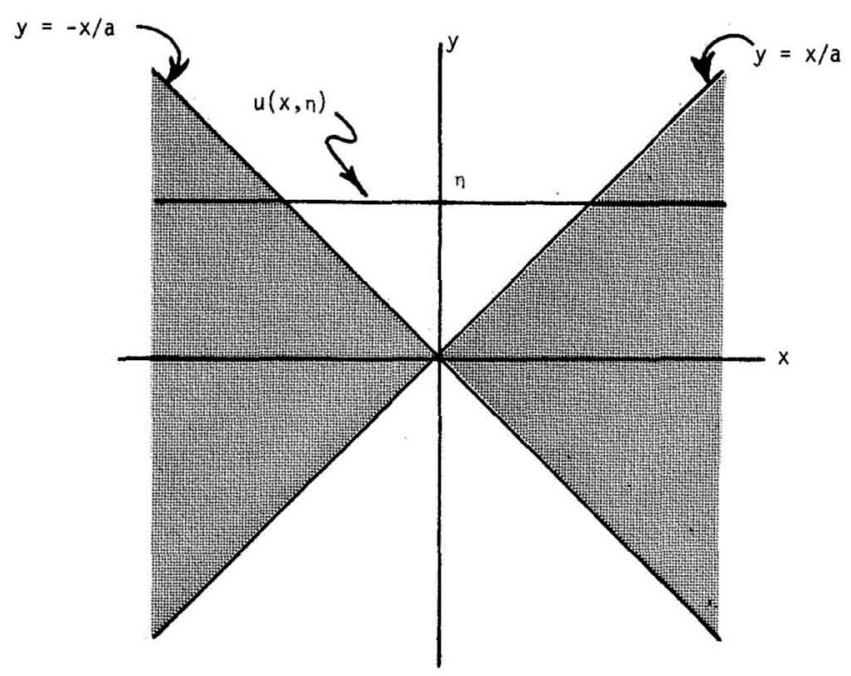

Fig. 1. The band-limited function $u(x, y)$ is known within the shaded cone. The extension problem is to evaluate the image in the unshaded cone. 


\section{References}

1. K. C. Tam and V. Perez-Mendez, "Tomographical imaging with limited angle input," J. Opt. Soc. Am. 71, 582 (1981).

2. T. Sato, S. J. Norton, M. Linzer, O. Ikeda, and M. Hirama, "Tomographic image reconstruction from limited projections using iterative revisions in image and transform spaces," Appl. Opt. 20, 395 (1981).

3. T. K. Sarkar, D. D. Wiener, and V. K. Jain, "Some mathematical considerations in dealing with the inverse problem," IEEE Trans. Antennas Propag. AP-29, 373 (1981).

4. D. C. Youla, "Generalized image restoration by method of alternating orthogonal projections," IEEE Trans. Circuits Syst. CAS-25, 694 (1978).

5. R. G. Wiley, "Concerning the recovery of a bandlimited signal or its spectrum from a finite segment," IEEE Trans. Commun. COM-27, 251 (1979).

6. G. A. Viano, "On the extrapolation of optical image data," J. Math. Phys. 17, 1160 (1976).

7. H. Stark, D. Cahana, and H. Webb, "Restoration of arbitrary finite-energy optical objects from limited spatial and spectral information," J. Opt. Soc. Am. 71, 635 (1981).

8. D. G. Luenberger, Optimization by Vector Space Methods (Wiley, New York, 1969).

9. R. J. Marks II, "Gerchberg's extrapolation algorithm in two dimensions," Appl. Opt. 20, 1815 (1981).
10. R. W. Gerchberg, "Super-resolution through error energy reduction," Opt. Acta 21, 709 (1974).

11. P. De Santis and F. Gori, "On an iterative method for super-resolution," Opt. Acta 22, 691 (1975).

12. A. Papoulis, "A new algorithm in spectral analysis and bandlimited signal extrapolation," IEEE Trans. Circuits Syst. CAS-22, 735 (1975).

13. A. Papoulis, Signal Analysis (McGraw-Hill, New York, 1977), pp. 244-248.

14. H. Stark, D. Cahana, and H. Webb, "Restoration of arbitrary finite-energy optical objects from limited spatial and spectral information," J. Opt. Soc. Am. 71, 635 (1981).

15. Ref. 4, Eqs. (11), (12), and (44) and Ref. 11.

16. R. J. Marks II and D. K. Smith, "Gerchberg-type linear deconvolution and extrapolation algorithms," in Transformations in Optical Signal Processing, W. T. Rhodes, J. R. Fienup, and B. E. A. Saleh, eds. (Society of PhotoOptical Instrumentation Engineers, Bellingham, Wash., in press).

17. D. Slepian and H. O. Pollak, "Prolate spheroidal wave functions: Fourier analysis and uncertainty I," Bell Syst. Tech. J. 40, 43 (1961).

18. D. Slepian and E. Sonnenblick, "Eigenvalues associated with prolate spheroidal wave functions of zero order," Bell Syst. Tech. J. 44, 1745 (1965). 